\title{
Meeting report 2005
}

Back in 2003 when we started planning the 2005 meeting of the American Aging Association, ('AGE'), we thought that the members and other attendees may be interested in a 'mix' of topics dealing with various aspects of MECHANISMS AND PREVENTION OF AGING. In keeping with this theme of the meeting, we selected speakers who would cover some of the most exciting recent developments in the study of the biology of aging as well as new information on the effects of nutrition and the prospects of nutritional interventions into the aging process in the human.

We were very gratified that the program appealed to many people with a near-record attendance of 239 . Approximately half of the participants arrived one day earlier to take advantage of the exciting PreConference program arranged by Dr. James Joseph, one of the past presidents of AGE and an internationally recognized expert on the influence of diet on oxidative stress and aging. The title of the PreConference, Nutrition, Lifestyles, Aging and Neurodegenerative Disease well describes the scope of very exciting findings which were presented and discussed during this part of the meeting.

The first session (Genetic Mechanisms - Chair: Dr. Trudy MacKay) provided an update of research on the mechanisms of aging in a worm, Caenorhabditis elegans, a fruit fly, Drosophila and yeast. These invertebrate organisms continue to play a key role in the study of genetic control of aging and the cellular mechanisms involved and allow application of the powerful novel molecular biology methods to the study of fundamental mechanisms of aging. (The speakers and titles of this and all other sessions as well as abstracts of their presentations can be found on the meeting website at http://www.americanaging. org/2005.html).

The second session (Cellular Mechanisms I Chair: Dr. Donald Ingram) dealt with age-related alterations in mitochondrial and muscle function and with recent advances in identifying changes in cellular metabolism that occur in response to caloric restriction.
The third session (Caloric Restriction in the Human - Chair: Claudio Franceschi) addressed the issue of the applicability of what we are learning about effects of caloric restriction in experimental animals to our own species. The speakers reviewed data obtained in individuals who voluntarily practice caloric restriction, findings from two on going controlled studies of caloric restriction in the human, and data relating nutrition to longevity to Okinawans, human population with the longest life-span.

The next session (Cellular Mechanisms II - Chair: Huber Warner) consisted of papers on DNA damage, relationship of growth hormone to aging, and phenotypic characteristics (including small body size) that predict longevity. This was followed by discussion of the role of IGF-1 in aging including data from two novel mouse models in which reduced levels or total of free IGF-1 are associated with extended life span (IGF-1 - Chair: Dr. Norman $W o l f$ ). Topics related to possibilities of treating age related disease and functional decline constituted the next session (Brain Aging and Novel Therapies Chair: Dr. John Morley). This included immunotherapy of Alzheimer's disease, role of stem cells in muscle regeneration, and protective effects of an anti-oxidant enzyme, SOD-1 on the survival of neurons.

The last session (Genomics and Proteomics Chair: Dr. John Holloszy) dealt with application of the powerful approaches of genomics and proteomics to analysis of the profiles of gene expression and gene products in different organisms.

There were also two sessions devoted to short presentations of recent research findings, selected from the abstracts submitted by the registrants. The high caliber of these presentations was most gratifying and I believe it attests to the appeal of the annual AGE meeting as a forum for reporting novel, exciting data. I should add that a total of 95 abstracts were submitted (11 oral, 79 poster) and those that were presented as posters were also excellent and contributed greatly to what the attendees learned during the meeting. 
In addition to the sessions listed above, there were three Special Lectures and an Award Lecture. Dr. Morley, director of the Gerontology Program at St. Louis University discussed the topic of frailty and reminded us that benefits of caloric restriction contrast with problems of loss of appetite, malnutrition and weight loss in the elderly. Dr. Franceschi, who developed an impressive series of studies of centenarians in Italy, reviewed the highlights of this fascinating work. Dr. Warner, who recently retired from a position of Program Director at the National Institute on Aging, reviewed the initiatives and achievements during the past 21 years. His lecture gave us a chance to express appreciation for what he did for the field of experimental gerontology and for the individual investigators and this was expressed in the introductory remarks made by Dr. Martin and in a standing ovation after the lecture. The Denham Harman Award Lecture dealt with mutations that prolong life in the mouse and included reflections of the undersigned on his personal journey from reproductive endocrinology to aging research.
I would like to thank all those who have made this meeting possible, including our generous supporters, as well as Drs. Peter Hornsby, Michal Jazwinski, Tom Johnson, George Roth, Holly Van Remmen, along with Dr. Arthur Balin - AGE Executive Director, Ms. Donna Cini - AGE Operations, the AGE Board of Directors and of course, all the speakers and meeting registrants!

We hope you will join us next year, once more, at our 2006 Annual Meeting to be held in Boston, Massachusetts (more details in our July issue). Until then, we look forward to your participation in our ongoing activities and hope that you will consider renewing your membership today (do not forget that each membership provides a considerable discount on the annual meeting registration as well as online access to our Journal)!

Andrzej Bartke, $\mathrm{PhD}$ AGE President, 2004-2005 